\title{
File System Performance Tuning for Standard Big Data Benchmarks
}

\author{
Da Qi Ren \\ Futurewei Technologies \\ 2330 Central EXPY, Santa Clara, CA \\ USA \\ daqi.ren@huawei.com
}

\author{
Bing Xia \\ Futurewei Technologies \\ 2330 Central EXPY, Santa Clara, CA \\ USA \\ bing.xia@huawei.com
}

\begin{abstract}
Modern file system manages super large data sets to perform data intensive and cost-effective analytical processing. Performance of a file system relies on storages, software, workload characteristic and configurations. Complex techniques have to be used in analysis because the data are often hybrid mix of different formats and different structured datasets. Performance study helps to optimize these factors and improve the design of a file system to meet the requirements of a specific application. A promising approach is to allocate the diverse data of various applications on different file systems according to their individual properties, in order to support the best possible performance to every particular application. Some basis that simulate the characters and scenarios of each step of data execution procedures are addressed in this paper. Based on workload characteristic analysis, administrator can implement tuning methods in the large and high-dimensional configuration parameter settings provided by the platform accordingly. Preliminary results are provided by running standard benchmark TPCX-HS, TPCx-BB, TPC-H and HiBench K-means on Ext4 and Btrfs file systems, and the impactions of workload characteristics to the benchmark performance have been analysed.
\end{abstract}

\section{CCS CONCEPTS}

Information storage systems $\rightarrow$ Storage architectures $\rightarrow$ Distributed storage $\cdot$ Software organization and properties $\rightarrow$ Extra-functional properties $\rightarrow$ Software performance

\section{KEYWORDS}

Big data, File system, Performance model, Benchmarks.

\section{INTRODUCTION}

Experiments show that each different file system (FS) is good at

Permission to make digital or hard copies of all or part of this work for personal or classroom use is granted without fee provided that copies are not made or distributed for profit or commercial advantage and that copies bear this notice and the full citation on the first page. Copyrights for components of this work owned by others than ACM must be honored. Abstracting with credit is permitted. To copy otherwise, or republish, to post on servers or to redistribute to lists, requires prior specific permission and/or a fee. Request permissions from Permissions@acm.org.

ICCDE 2018, May 4-6, 2018, Shanghai, China

(C) 2018 Association for Computing Machinery.

ACM ISBN 978-1-4503-6393-8/18/05...\$15.00

https://doi.org/10.1145/3219788.3219809 a different set of workloads with different characteristics. [1] Big data workloads have many different characteristics including file popularity, temporal locality, request arrival pattern and data access pattern. An architect can build big data platforms using a plenty of very different file systems, but it is important to find the best suited FS to accomplish the specific requirement for each individual. FS performances are characterized by disk $\mathrm{read} /$ write bandwidth, I/O device utilization, average waiting time of $\mathrm{I} / \mathrm{O}$ requests and average size of $\mathrm{I} / \mathrm{O}$ requests. MapReduce paradigm and Hadoop HDFS are current fundamental architecture for big data processes. Most of MapReduce and HDFS works have similar file usage patterns. At upper software layer, library stacks and programming languages such as Spark, Hive, Impala and user threads also impact the performance of storage and file system.

An approach is proposed in this work to analyze the scenario basis of workloads executed across different layers of platform, then based on the characteristics of program algorithms and the run-time behaviors of workloads to assign the data files onto different file system. The throughput of each layer is modelled by deploying appropriate parameters to predict the FS impact to the workload behaviors. Following this idea, a table is made based on dynamic tunable features provided by big data platform and is applicable to both upper application and database systems. Various systems have been studied including enterprise storage systems, web servers, clusters and network systems. Workloads have also been analyzed at different levels: such as workload characterizations; [2] [3] Job execution patterns, data storages and I/O performance; [4] and the distribution data set and overall resource utilizations. [5] Some studies have provided with metrics about data access pattern in MapReduce scenarios [4], but these metrics are limited. [4]

\section{FILE SYSTEM AND PERFORMANCE FACTORS}

Popular FS systems being used in data centers include Ext3, Ext4, Xfs, Btrfs (linux OS) and FAT, NTFS, exFAT (windows OS) [1]. The above file systems have very different features that can contribute a same program different performances. Linux FS performances are compared in [6] by using benchmark tests with basic operations including sequential/random read/write for characters and blocks; random seeks and file compressions; semi-synthetic read/write speed and database operations. The performance differences are summarized in Table 1. [6] It shows each file system performed the best at some key fronts of different usage patterns. 


\subsection{Hadoop File System}

HDFS stores data on cluster machines connected with high aggregate bandwidth. Utilization of HDFS follows a certain pattern that can be described respect to the disk and network I/O incurred through the Hadoop MapReduce job process. In detail, a Hadoop job starts with a Job configuration object, gets the job-id from the job tracker and writes the job configuration object and

Table 1. Benchmark Results [6]

\begin{tabular}{l|l|l|l|l}
\hline \multicolumn{1}{c|}{ Functions } & \multicolumn{4}{l}{ Performance ( Fair $\rightarrow$ Best) } \\
\hline seq. char in & Ext4 & Ext3 & Xfs & Btrfs \\
seq. char out & Xfs & Ext4 & EXT3 & Btrfs \\
seq. block In & Ext4 & Xfs & EXT3 & Btrfs \\
seq. block out & Ext4 & Xfs & Btrfs & Ext3 \\
seq. block direct in & Ext4 & Xfs & Est3 & Btrfs \\
seq. block direct out & Ext4 & Ext3 & Btrfs & Ext3 \\
random seeks & Ext3 & Xfs & Ext4 & Btrfs \\
file create & Ext4 & Xfs & Ext3 & Btrfs \\
file remove & Ext4 & Xfs & Ext3 & Btrfs \\
seq. read & Xfs & Ext4 & Ext3 & Btrfs \\
seq. write & Ext4 & Xfs & Btrfs & Ext3 \\
random read & Xfs & Btrfs & Ext3 & Ext4 \\
random write & Ext3 & Xfs & Ext4 & Btrfs \\
prep.100K r PSQL & Btrfs & Ext3 & Ext4 & Xfs \\
rd.100K r PSQL & Ext3 & Xfs & Ext4 & Btrfs \\
complex R/W /trans & Ext3 & Xfs & Ext4 & Btrfs \\
Linux untar & Xfs & Btrfs & Ext4 & Ext3 \\
Linux tar & Xfs & Ext3 & Ext4 & Btrfs \\
seq wt. fragment & Ext4 & Xfs & Ext3 & Btrfs \\
rand wt. fragment & Ext4 & Xfs & Ext3 & Btrfs \\
\hline
\end{tabular}

related information to the job tracker's directory. The input format class is also instantiated and written to this directory along with Input Splits. The mapper takes data from the HDFS system and the data flushed onto the disk from multiple mappers all incur the disk I/O. The reducer processes and the shuffled data in the HDFS read from the disk by the reducer will cause disk I/O. This data blocks are replicated at many other Data Nodes of HDFS for multiple jobs and subsequent jobs, that will again lead to additional disk I/O. Performance factors locates in each layer of the platform. Storage on Data Node limits how much concurrent processing a node can support at the same time. The number of task slots has no effects on I/O metrics, but increasing the number of task slots appropriately can accelerate the execution speed. Increasing memory can reduce the number of I/O requests, alleviate the pressure of disk read/write, and effectively improve the $\mathrm{I} / \mathrm{O}$ performance when the data size is large. The compression of intermediate data mainly affects the MapReduce I/O performance and has little influence on HDFS I/O performance. HDFS's I/O pattern is large sequential access and MapReduce's I/O pattern is small random access. When configuring storage systems, the number of devices and the types of devices are important to take account. HDFS performance factors are collected in Table 2.

\subsection{BTR File System vs. EXT4 System}

Both Ext4 and BTR are file systems being popular used in Linux OS. Ext4 uses extents, i.e. a contiguous group of blocks, makes allocation storage much more efficient. It decreases fragmentation and support much larger files and volumes, i.e. the maximal file size in Ext4 is $16 \mathrm{~TB}$, whereas the maximum volume size is even set to $1 \mathrm{~EB}$. Ext4 is accomplished with preallocation features and delayed allocation features, i.e. blocks can be reserved but not used immediately and the actual writing of files can be delayed until the last possible moment, which simultaneously increases performance and decreases fragmentation.

Btrfs is B-tree File System designed to become the next major Linux file system to offer better scalability and reliability and can be expected to replace Ext4 in the future [7]. It is a copy-onwrite file system primary focus points include fault tolerance, repair, and easy administration. The main features are: Shrink and grow storage volumes; Copy-on-Write; extensive checksums maintained for all metadata and data; efficient writeable snapshots, clones as first class citizens; multi-device support; online resize and defragmentation; space-efficient packing of small files and many more useful improvements ensure data integrity.

\section{METHODOLOGY}

\subsection{Methodology}

The method proposes to create a table that can map workload characteristics of each application to a set of FS attributes and settings. Based on this table, Name ode can dynamically manage database distribution adapting to the FS environmental attributes. When an application saves a data file, Name Node would be able identify the proper data storage format to use, e.g. E4 (EXT4), E3 (EXT3), B (BTRFS) or other arguments for other formats. The table is designed to save each Data Node's storage formats in "Storage Format" list; a data type of "Storage Format" is added to the Name Node where the Data Node Information is stored. Name Node collects "Storage Format" information from each Data Node periodically, and update the corresponded storage information in Name Node. When Data Node is reformatted, the storage information will be reported to the Name Node. By default, the secondary Name Node information will be updated accordingly.

While a data file is being saved to HDFS, the Name Node will firstly search all "free" Data Nodes and disks by checking the storage format table to find where has the most suitable FS format. If there doesn't have node or disk with matched format, the Name Node use default system strategy to save the file. If there is a format matching node and disk is found, save the file on the disk, and update the status to log file and the user.

While HDFS balancer perform data balancing process, it will firstly check the original "Storage Format Table" of the data block to be transferred. After the HDFS located all "free nodes", check the Storage format Table to find what node having the same format as the original data block. If there is no a matchformat node is found, the Name Node use default system strategy to save the file. Otherwise, save the data block to a 
matched format node, and send the status to $\log$ file and the user.

While a HDFS runs a checkpoint process to store the latest updates and new edits, it will backup all files edited since the previous checkpoint and merge them into a new FSimage. Name node saves the new FSimage files using the same storage format as the last FSimage. While the HDFS located all "free nodes", it checks the "Storage Format" table to find what node having the same format as the original FSimage. If there is no a matchformat node is found, ignore this request and use system default strategy to save the FSimage. If there are matched format nodes and disks are found, Name Node saves the FSimage and send the status to $\log$ file and the user.

\subsection{Benchmark}

To validate this method, standard benchmarks of TPC-HS, TPC$\mathrm{BB}, \mathrm{TPC}-\mathrm{H}$ and $\mathrm{K}-$ means clustering (in HiBench) have been used. Each one of them is composed of theoretical and real world test inside, and provides methods for comparing the performance of various big data platforms across different architectures. They measures processor and memory performance, disk and network $\mathrm{I} / \mathrm{O}$, scalability of building distributed applications, and usage of the system resource [8]-[10].

\section{ENVIRONMENT AND BENCHMARK}

\subsection{Workload}

\subsubsection{K-means Clustering}

K-means Clustering is a clustering algorithm for knowledge discovery and data mining. Given a set of samples where each one is represented as a d-dimensional vector, the algorithm find the centroid and each cluster by running Hadoop job iteratively, until different iterations converge. It has two stages, namely iteration (CPU-bound) and clustering (I/O-bound). In this work, the open source code K-means in HiBench Benchmark kit is used.

\subsubsection{TPCX-HS}

TPC Express Benchmark HS (TPCx-HS) is developed using Hadoop sort to provide an objective measure of hardware, operating system and commercial FS distributions. [10] It is recognized by the industry with verifiable performance, priceperformance and availability metrics. Hadoop sort is both CPU and $\mathrm{I} / \mathrm{O}$ intensive.

\subsubsection{TPC-H}

TPC-H is developed using database queries for providing an objective measure of high sequential I/O-throughput (large I/O operations). It is critical to excel in large hash-join operations. TPC-H implements both single- and multi-user tests. [9]

\subsubsection{TPCX-BB}

TPCX-BB uses 30 pre-defined analytics queries to simulate real world conditions in a specific context -- namely, that of a retailer with a combined online and brick-and-mortar presence. Queries consist of a mix of SQL statements (expressed in SQL, passed to Hive or Spark) and machine learning (ML) algorithms (using ML libraries, user-defined functions, and procedural code). TPCX-BB measures the performance of Hadoop-based systems. It also specifically refers to Hive or Spark for SQL query processing. The workload is mixed CPU-bound workload and I/O bound workloads. [8]

\subsection{Platform}

Six Tecal RH2288 V2 Rack servers have been used in this work, each with 2 Intel Xeon Processor E5-2680 (Sandy Bridge-EP) running at $2.7 \mathrm{GHz}$. Each processor has 8 cores (16 hyperthreaded) and a 20MB L3 cache. They are connected through two QuickPath links, each providing a unidirectional transmission rate of up to $8.0 \mathrm{GT} / \mathrm{s}$. Each server has $248 \mathrm{~GB}$ double data rate 3 (DDR3) at $1066 \mathrm{Mhz}$ dimms of main memory with a total of $192 \mathrm{~GB}$. Each server is configured with eight

Table 2. Performance factors in each layer

\begin{tabular}{|c|c|}
\hline Hierarchy & Performance Factors \\
\hline $\begin{array}{l}\text { Algorithm } \\
\text { and } \\
\text { Implement } \\
\text { ation }\end{array}$ & $\begin{array}{l}\text { O Stress points } \\
\circ \text { I/O utilization } \\
\circ \text { Operations type ( e.g. query, join) } \\
\circ \text { Recursive iterations } \\
\circ \text { CPU usage and bound } \\
\circ \text { Cluster architecture }\end{array}$ \\
\hline $\begin{array}{l}\text { Software } \\
\text { Platform }\end{array}$ & $\begin{array}{l}\text { o Average I/O data size of a map task } \\
\text { o Ratio of a map output to a map input } \\
\text { o Total number of map/reduce tasks/slots } \\
\text { o Average execution time of a map/reduce task } \\
\text { o Number and size of shuffle tasks in each wave }\end{array}$ \\
\hline OS & $\begin{array}{l}\circ \text { Type of FS system ( e.g. HDFS) } \\
\circ \text { I/O access pattern } \\
\circ \text { File compressions } \\
\circ \text { CPU Hyper threading }\end{array}$ \\
\hline Hardware & $\begin{array}{l}\text { Memory Size }(\mathrm{GB}) \\
\circ \text { Storage Size }(\mathrm{GB}) \\
\circ \text { I/O performance } \\
\circ \text { Storage Format }\end{array}$ \\
\hline
\end{tabular}




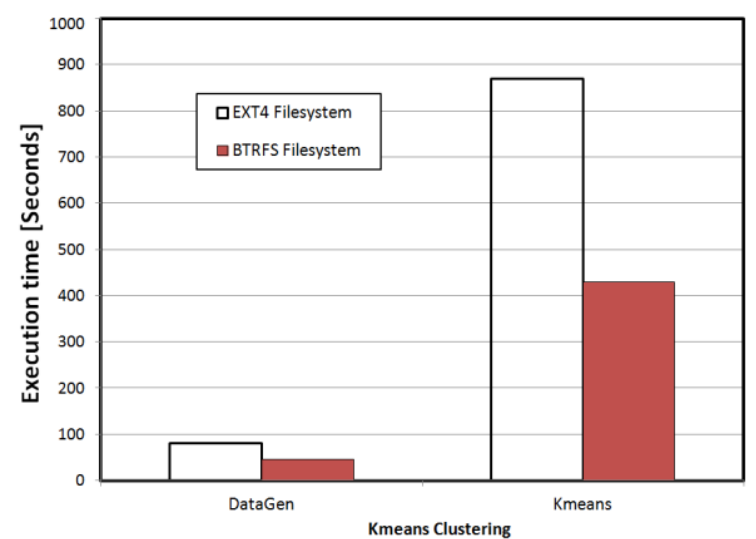

Figure 1: A performance comparison for HiBench K-means clustering benchmark executed on both Ext4 and Btrfs file systems.

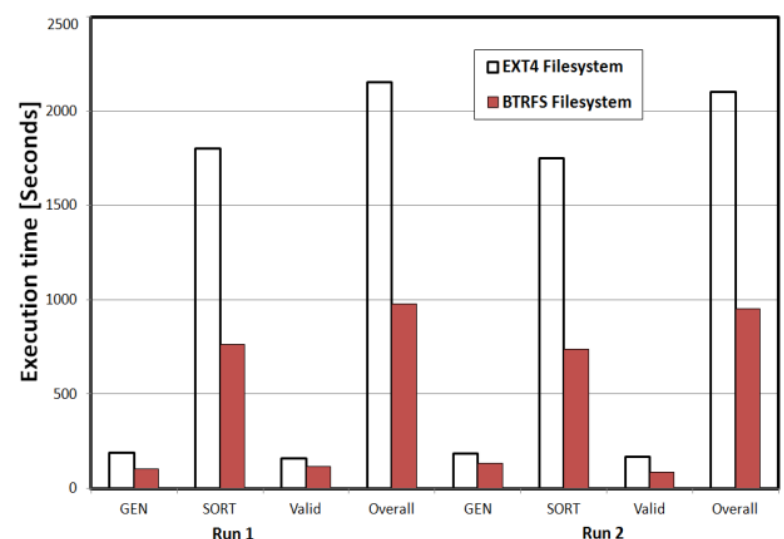

Figure 2: A performance comparison for TPCx-HS benchmark executed on both Ext4 and Btrfs file system.
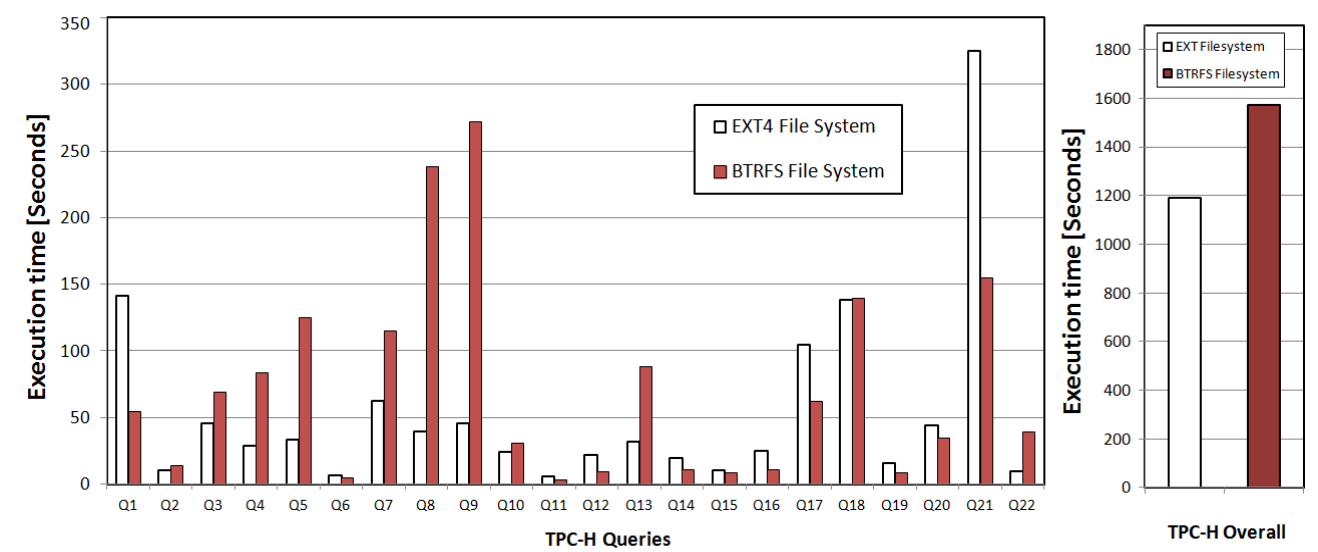

Figure 3: A performance comparison for TPC-H benchmark power run executed on both Ext4 and Btrfs file systems.
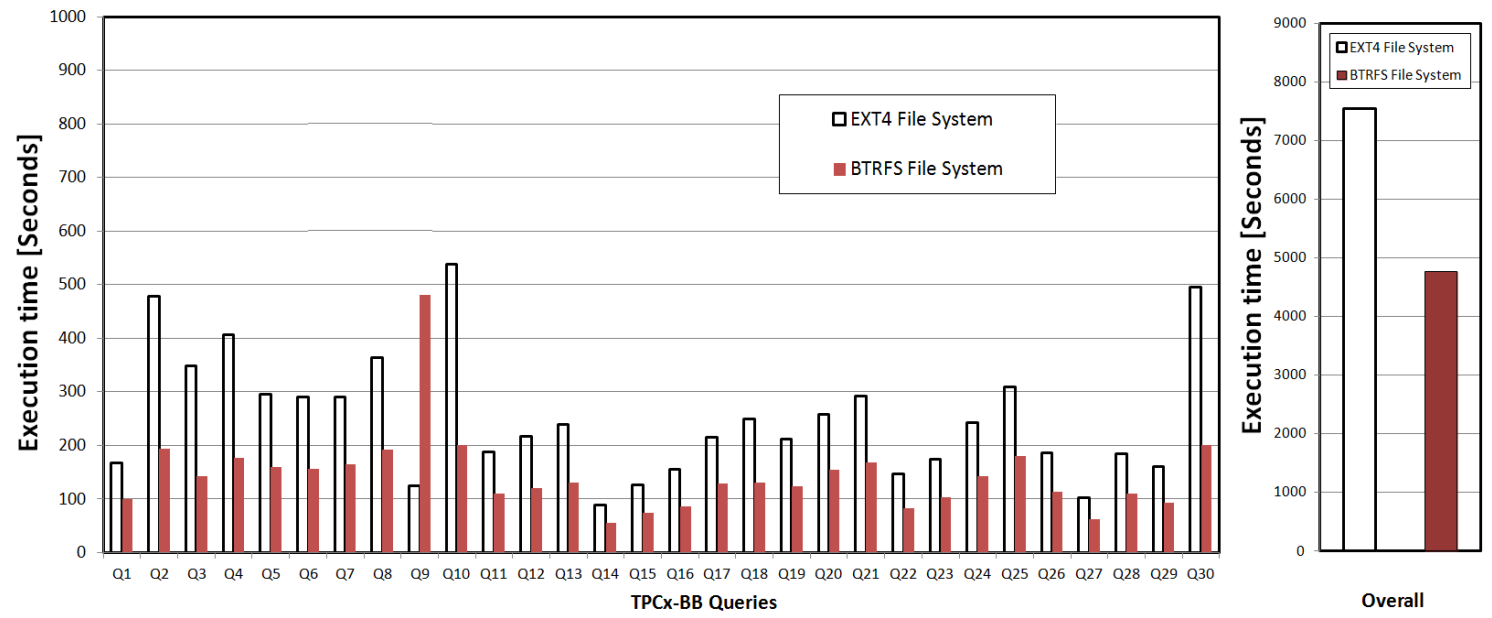

Figure 4: A performance comparison for TPCx-BB benchmark power run executed on both Ext4 and Btrfs file systems. 
2.5" SAS HDDs with 7.2TB capacity. One SAS disk hosts the OS and the remaining 7 are configured for HDFS. The server provides four onboard gigabit Ethernet (GE) ports, of which two were bonded to double bandwidth. The system runs CDH 5 on centos 6.5. Cloudera is configured as one master node and five worker nodes, Apache Hadoop 2.6 is installed. Each of the nodes has 189.1GBytes of memory. The same hard drives were firstly formatted in Ext4 format, then Btrfs format, for performance comparison on the same machines. All the other software and hardware environment are identical in both tests.

\section{Experiments}

\subsection{Results}

\subsubsection{K-means Clustering}

The size of the initial data set is $100 \mathrm{G}$ in the K-means clustering benchmark. Firstly the program generates initial data set on the disks; secondly the clustering calculations are processed. The performance measurements of both steps are shown in Figure. 1. At the data generation stage, Btrfs is $45.2 \%$ faster than Ext4 file system; at the clustering stage, Btrfs is $50.6 \%$ faster than EXT file system.

\subsubsection{TPCX-HS}

A full TeraSort benchmark run consists of the three phases: 1) generating the input data via TeraGen; 2) running TeraSort on the input data; data; 3) validating the sorted output data via TeraValidate. The performance comparison of TPCx-HS is shown in Figure.2. Taking average of performances in Run 1 and Run 2, Btrfs file system has improvements of $37.65 \%$ at data generation stage; $57.78 \%$ at data sorting stage, and $39.03 \%$ at data validation stage. In total, Btrfs performs $54.5 \%$ faster on overall execution time comparing with Ext4 file system.

\subsubsection{TPC-H}

TPC-H benchmark give equal importance to the single- and multi-user runs. The performance comparison of TPC-H is shown in Figure. 3. We find in the queries Q1, Q6, Q11, Q12, Q14, Q15, Q16, Q17, Q19, Q20, Q21 Btrfs perform better than Ext4; and in the rest of the queries, Ext4 perform better than Btrfs. The overall performance of Ext 4 is $32.6 \%$ greater than Btrfs.

\subsubsection{TPCX-BB}

TPCX-BB benchmark includes 3 phases: 1) Load test: to test how fast raw data can be read from the distributed file system; 2) Power test: to test the system using short-running jobs with less demand on cluster resources, and long-running jobs with high demand on resources; 3) Throughput test: to test the efficiency of cluster resources by simulating a mix of short and longrunning job executed in parallel. The performance comparison of TPCX-BB is shown in Figure. 4. In general, the performance of Btrfs file system is better than Ext4 file system at almost all queries except Q9. The overall performance of Btrfs is greater than Ext4 by $46.5 \%$ percent.

\subsection{Analysis}

Two file systems have been chosen to compare, i.e. Ext4 and Btrfs. Btrfs features a number of file system improvements including the compression of intermediate data that mainly improves the MapReduce I/O performance, while consumes some CPU resource which may influence the job's execution time. The I/O pattern of HDFS and MapReduce are different, HDFS's I/O pattern is large sequential access and MapReduce's $\mathrm{I} / \mathrm{O}$ pattern is small random access. TPCX-HS, TPCX-BB and Kmeans Clustering benchmark are all $\mathrm{I} / \mathrm{O}$ intensive and CPU intensive, they can work better with Btrfs. Workload like TPCX$\mathrm{H}$ is $\mathrm{I} / \mathrm{O}$ intensive, it can work better with Ext4 system.

\section{CONCLUSION AND FUTUREWORK}

A method has been proposed to map workload characteristics to suitable file systems for improving FS performance. To validate this method, standard benchmarks have been used to investigate the characters of workloads, and the corresponding performance measures on different FS. Specifically, we use standard benchmarks TPCx-BB, TPCx-HS, TPCx-H and HiBench to test on the real platform of Ext4 and Btrfs FS. A preliminary model is provided that can help to handle performance factors in order to improving the design and configurations to meet the application needs. Higher level software design and algorithm determines the actual workflow execution on each level of the system, and decide the pattern of resource usage. Better understand to workload characters and the feather of the big data system are both important to performance researchers.

\section{REFERENCES}

[1] Comprison of file systems, https://en.wikipedia.org/wiki/Comparison_of_file_systems

[2] Parmita Mehta, Sven Dorkenwald, et al. 2017. Comparative Evaluation of BigData Systems on Scientific Image Analytics Workloads. In Proceedings of the VLDB Endowment, Vol. 10, No. 11.

[3] N. Peters, S. Park and S. Chakraborty. 2016. Web browser workload characterization for power management on HMP platforms. In Intl. Conf. on Hardware/Software Codesign and System Synthesis, Pittsburgh, PA, USA.

[4] K. Ren, G. Gibson, Y. Kwon, M. Balazinska, and B. Howe. 2012. Hadoop's Adolescence; a Comparative Workloads Analysis from Three Research Clusters. In Proc. SC Companion.

[5] J. Wang, X. Zhang and J. Yin. 2016. Speed Up Big Data Analytics by Unveiling the Storage Distribution of Sub-datasets. IEEE Transactions on Big Data.

[6] Jonatan Dante, http://www.ilsistemista.net/index.php/linux-a-unix/6linux-filesystems-benchmarked-ext3-vs-ext4-vs-xfs-vsbtrfs.html?limitstart $=0$

[7] Ext4 file system source: https://en.wikipedia.org/wiki/Ext4

[8] TPCx-BB source: http://www.tpc.org/tpc_documents_current_versions /current_specifications.asp

[9] M. Poess, D. Ren, T. Rabl, H. JacobsenHans. 2018. Methods for Quantifying Energy Consumption in TPC-H, In ACM/SPEC International Conference. Berlin, Germany.

[10] R. Nambiar, M. Poess, D. Ren, et al. 2014. Introducing TPCx-HS: Industry's First Standard for Benchmarking Big Data Systems. Performance Characterization and Benchmarking. Traditional to Big Data, DOI 10.1007/978-3-319-15350-6 